\title{
Dual tracer imaging approach in assessing tumor biology and heterogeneity in neuroendocrine tumors: its correlation with tumor proliferation index and possible multifaceted implications for personalized clinical management decisions, with focus on PRRT
}

\author{
Sandip Basu • Bhawna Sirohi $\cdot$ Shailesh V. Shrikhande
}

Received: 14 March 2014 / Accepted: 5 May 2014 / Published online: 27 May 2014

(C) Springer-Verlag Berlin Heidelberg 2014

The recent years have witnessed a rapid expansion and application of functional somatostatin receptor-targeted imaging and therapy in the clinical management of metastatic or inoperable neuroendocrine tumors (NETs). This approach now plays a pivotal role in the clinical decision making of this group of patients, given the highly targeted and effective nature of peptide receptor radionuclide therapy (PRRT) in tumors with substantial somatostatin cell surface receptor expression and its noted tolerability with minimal side effects. The popularity of the therapeutic option has steadily increased and is now frequently considered among the multimodal management strategies that include [1, 2]: (i) Cytoreductive surgery (if feasible), (ii) medical therapy (encompasses cold somatostatin analogs, targeted agents and chemotherapy), (iii) locoregional ablative procedures such as radiofrequency ablation (RFA), selective hepatic transcatheter arterial embolization (TAE), chemoembolization (TACE), selective internal radiotherapy (SIRT), and laser-induced thermotherapy (LITT). One of the salient decision-making factors for choosing the appropriate therapy has been the tumor grade, which is based upon the proliferation index (also known as Ki-67 labeling index, a marker for cellular proliferation). Some of

S. Basu $(\bowtie)$

Radiation Medicine Centre, Bhabha Atomic Research Centre,

Tata Memorial Hospital Annexe, Jerbai Wadia Road, Parel,

Mumbai 400 012, India

e-mail: drsanb@yahoo.com

B. Sirohi

Dept of Medical Oncology, Tata Memorial Hospital, Mumbai, India

\section{S. V. Shrikhande}

Department of Surgical Oncology, Gastrointestinal and

Hepato-Pancreato-Biliary Service, Tata Memorial Hospital, Mumbai, India the reasons PRRT is preferred over the newer targeted agents available (everolimus and sunitinib) include the cost of these new drugs, toxicity, and the need to remain on these drugs until progression, while PRRT is a one-off procedure completed in few sittings. In this article, we explore how functional molecular imaging correlates with the histopathological characteristics of a tumor and whether patient-specific, personalized medicine can be better employed in the management of gastroenteropancreatic neuroendocrine tumors (GEPNET), based upon functional imaging information. Ideally, this imaging would provide a global picture of the lesions and the disease, contributing to selection of the correct treatment option and helping to define the disease prognosis.

\section{Tumor proliferation index and NETs: implications} for clinical management decisions in metastatic NETs

Histological tumor grading, along with stage and age, are strong predictors of overall outcome in NETs, of which the former is of pivotal importance in prognostic risk stratification and has been frequently utilized for treatment decisionmaking. Irrespective of the endeavors and different systems of classifications by various groups, the Ki-67 labeling index (LI; depicting Ki-67-positive tumor cells, the fraction that are in the proliferative phases of the cell cycle, i.e., $G_{1}, S, G_{2}$, and mitosis) has been the major determinant of histological grading of NETs [3-9]. In the practical scenario, attending physicians are better versed with the MIB-1 labeling index (more commonly observed in histopathology reports, and has essentially replaced the original Ki-67 index in practice), which is directed against different epitopes of the same proliferationrelated antigen MKI67 and has the advantage of being estimated on formalin-fixed, paraffin-embedded sections. Current 
guidelines (ENETS, WHO), of course, refer to the Ki-67 index as the standard nomenclature, assessed in 2,000 tumor cells in areas having the highest nuclear labeling $[6,7]$.

The standard indication of peptide receptor radionuclide therapy (PRRT) with radiolabeled somatostatin receptor analogues $\left({ }^{177} \mathrm{Lu} /{ }^{90} \mathrm{Y}\right.$-labelled DOTA-TOC/ TATE) has been advanced/metastatic/inoperable welldifferentiated grade 1 and 2 NETs (this corresponds to Mib1 (Ki-67) LI of up to $20 \%$ ) that express somatostatin receptor positivity, evaluated by somatostatin receptor-based imaging (SRI) with 68Ga-DOTA-TOC/ TATE/NOC PET-CT or 99mTc-HYNIC-TOC scintigraphy or 111 In-octreoscan $[1,2,10]$. The former imaging approach is preferable due to its superior resolution and hence, better sensitivity and the advantage of quantification. The latter approach, along with SPECT imaging, can be conveniently employed in centers that do not possess the $68 \mathrm{Ge}-68 \mathrm{Ga}$ generator or that do not have a PET-CT facility [11]. Chemotherapy is the preferred option in high-grade (G3) neuroendocrine carcinoma (NEC), with increasing proliferation index more preference towards the combination chemotherapeutic approach is given because of better chemosensitivity of the tumor $[1,2,10,12]$.

\section{Functional molecular imaging and MiB 1 index: the correlation}

Both somatostatin receptor-targeted molecular imaging (SRI) and FDG PET/CT imaging have been utilized for the evaluation of NETs, though the former is the major decision-making scan used for evaluating somatostatin receptor positivity of the lesions and deciding upon the suitability of PRRT. On a graded visual analysis of the diagnostic somatostatin receptor-targeted imaging, tracer uptake in metastatic lesions is a more important imaging determinant than the normal hepatic uptake for selecting the patient for PRRT [10, 12-14]. FDG-PET/CT, on the other hand, assesses glycolytic metabolism, and its higher uptake is associated with tumor aggressiveness [15]. A dichotomous behavior exists between both of these tracers in well-differentiated and poorly differentiated NETs [16, 17], where the former is more positive with somatostatin-targeted imaging and the latter with FDG-PET/CT. Hence, a combination dual-tracer approach encompassing somatostatin receptor and FDG PET imaging assessing the somatostatin receptor cell surface (SSTR) expression and glycolytic metabolism, respectively, can be utilized to enhance treatment selection as well as disease prognosis in a more scientific manner.

The possible clinical implications of dual-tracer molecular imaging vis-a-vis the tumor proliferation index: will the former take the upper hand for prognosis and guiding individualized therapy?

We herein enumerate the possible clinical areas where the dual-tracer imaging information can be potentially useful to augment individualized clinical decision making in NET with an emphasis on PRRT.

[a] Assessing disease biology on a continuous scale at intermediate Mib1 (Ki-67) indices:

While total discordance between the two tracers at extremes of tumor differentiation is the usual observation, they are not so clear-cut at the intermediate indices. We have
Fig 1 SRI with 99mTc-HYNICTOC and FDG-PET/CT in a 59yr-old male; postoperative case of NET of the head and body of the pancreas presenting with recurrent, heterogeneously enhancing paraduodenal mass with liver metastasis. The Mib1 LI was reported to be $1-2 \%$ and the serum $\mathrm{Cg}$ A was $1,375 \mathrm{ng} / \mathrm{ml}$. The recurrent mass and the hepatic lesion showed high-grade uptake in SRI but negligible uptake on PET-CT (i.e., total discordance between SRI and FDG-PET/CT)

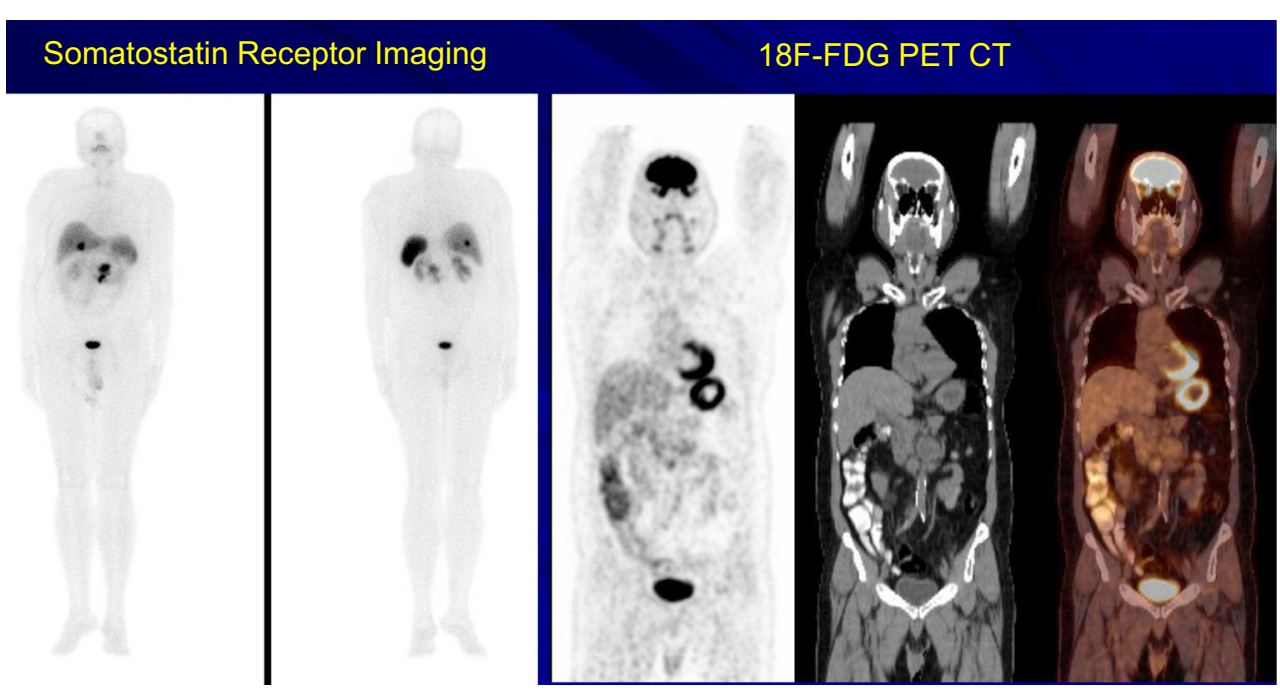


Fig 2 A 60-yr-old male, presented with a $11 \times 9 \times 11-\mathrm{cm}$ heterogeneous mass arising from the pancreatic body and tail with liver metastases; Mib1 index of the primary: $4 \%$. A partial concordance was observed between SRI and FDG-PET/CT, both in the primary and at the metastatic lesions. Both intralesional (in the primary) and interlesional heterogeneity (amongst the hepatic metastases) are easily observed in the images with regard to FDG avidity and positivity

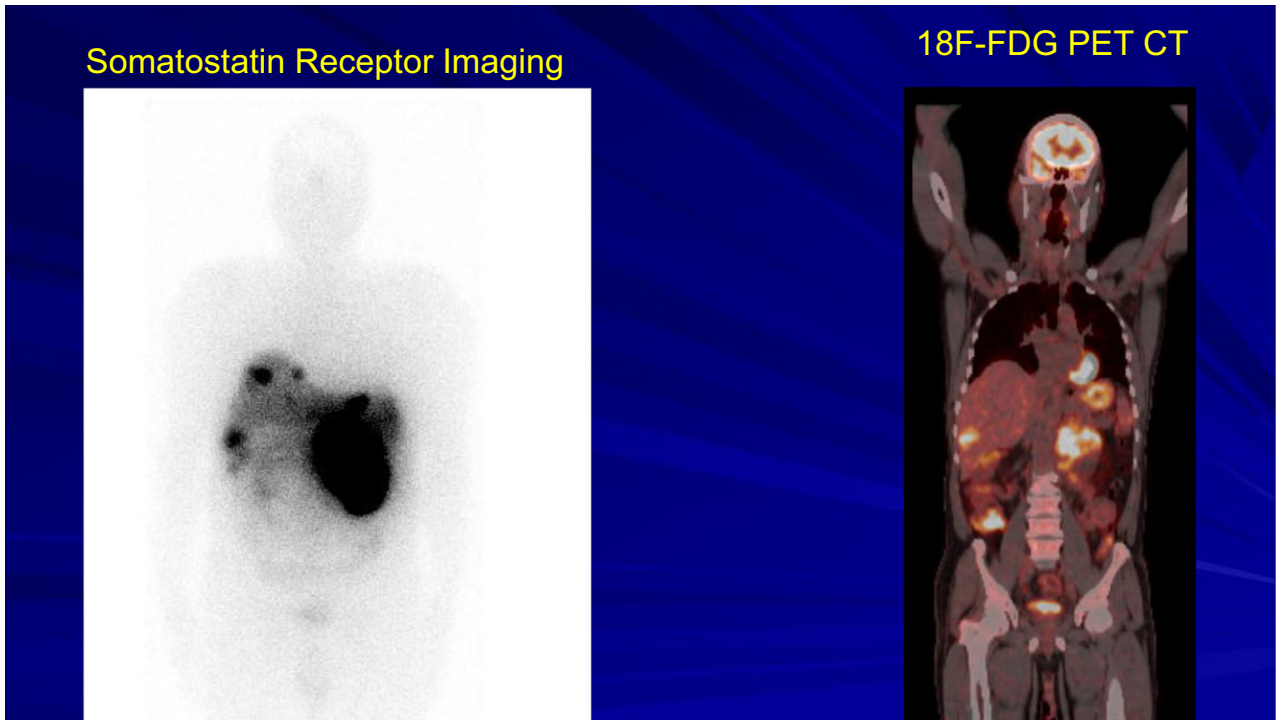

observed in our practice (in patients who had undergone PRRT), that while most patients with less than $2 \% \mathrm{Mib}$ 1 LI demonstrated a near-total discordance with high somatostatin receptor positivity and little or absent FDG activity, the uptake and the lesion positivity on FDG increased with increasing Mib1 (Ki-67) LI (partial concordance between FDG and SRI). Total concordance of the lesions was observed in virtually all lesions above Mib1 (Ki-67) LI of $15 \%$ (unpublished data). However, discordance between the imaging results and proliferation index does exist, in which case, the in-vivo imaging shows discrete advantages compared to the histopathology alone (addressed below, separately). Thus, when correlated, the molecular imaging with the dual-tracer approach has the potential to show the overall biology on a continuum where high FDG avidity of a lesion signifies poorer prognosis (Figs. 1, 2, and 3).

[b] Assessing intra- and interlesional heterogeneity in metastatic lesions:

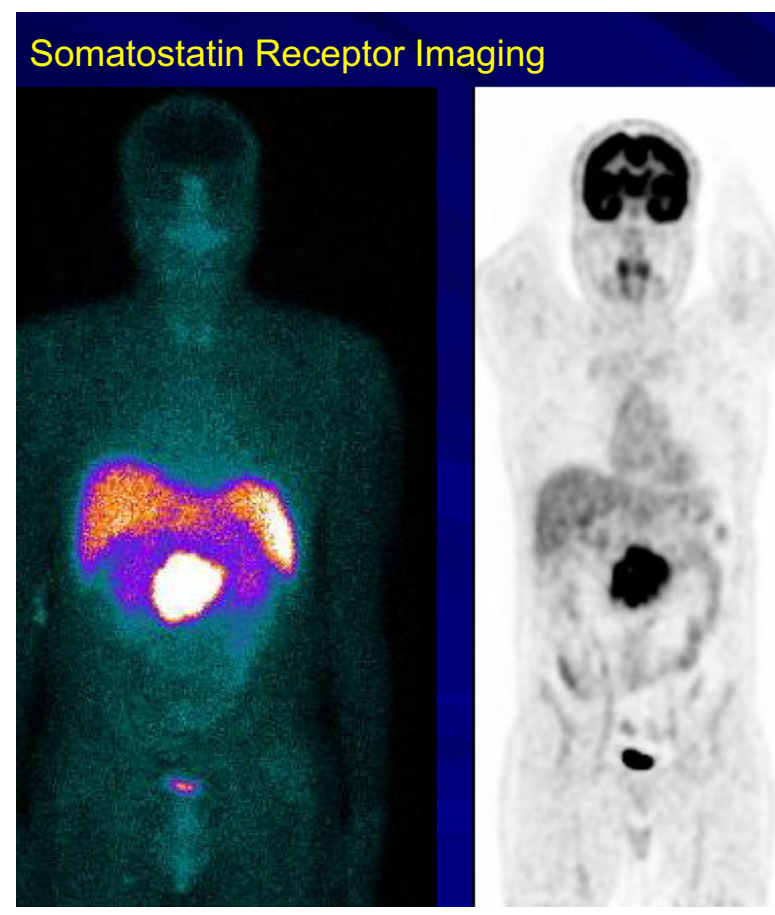

Fig 3 A 44-yr-old male, presenting with a 6.1 x 6.3 x 4-cm highly vascular mass in the uncinate process of the pancreas with duodenal and SMA infiltration on ceCT. The histopathology report suggested

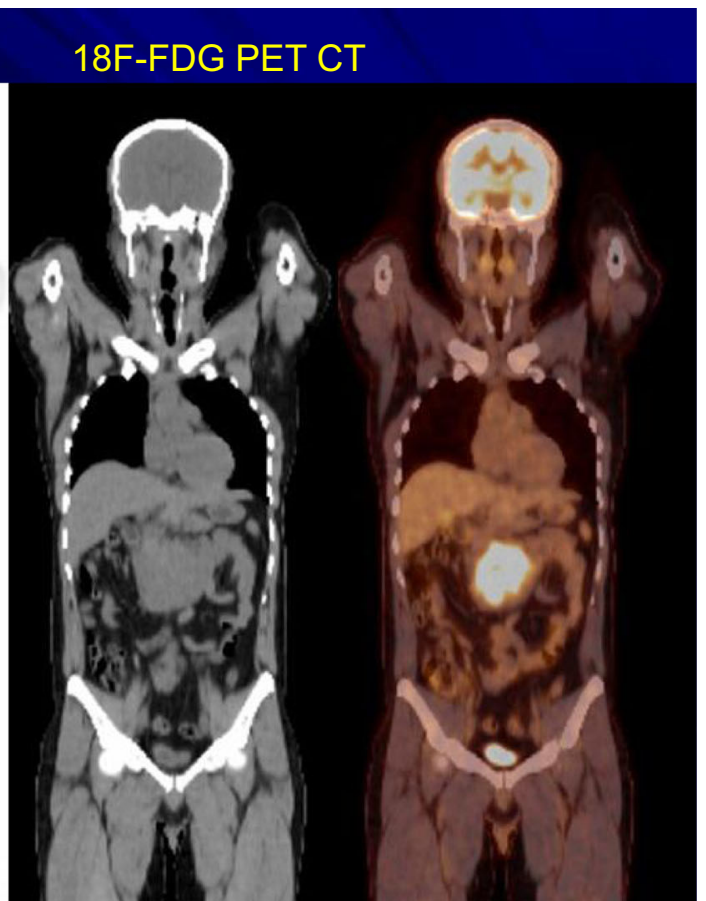

poorly-differentiated NE carcinoma with Mib1 LI -30\%. A concordance is observed between SRI and FDG-PET/CT, with the mass having FDG avidity consistent with its proliferation index 
The ability to perform the imaging assessment as a whole-body study is a discrete advantage compared to single-site biopsy. Both intra- and intertumoral heterogeneity are known to exist in cancer [17], and the same can be encountered in NETs as well. Thus, in the same individual, different lesions (or different parts of a same large lesion) can demonstrate different grades of tracer uptake indicating different tumor biology, and hence different aggressiveness. Thus, high FDG uptake would suggest an aggressive behavior and the possibility of treatment refractoriness of the cells at the site, whereas low uptake would indicate a biologically indolent lesion (Fig. 2). In the scenario of ongoing treatment, appearance and increase of FDG uptake in a particular lesion among several lesions would suggest the development of treatment resistant clones at that site.

[c] Discordance between molecular imaging and tumor proliferation index of the primary

The intralesional clonal heterogeneity in the primary can, at times, lead to discordance between the molecular imaging characteristics and the Mib1 (Ki-67) index obtained. The limitations of relying on single-site histopathology should be kept in mind when one encounters such discordance, where the molecular imaging would be representative of the global phenotype of the tumor, thus predicting the treatment outcome more appropriately. (Figure 4).

[d] Treatment decision-making in Mib1 (Ki-67) LI between 20 and $30 \%$ : can the dual-tracer approach help in individualization?
With the increasing popularity and reliance on PRRT among the medical oncology community, efforts have been made to extend its indications beyond the suggested limit of Mib1 (Ki-67) LI of $20 \%$ [2, 18]. Thus, though the joint IAEA, EANM, and SNMMI practical guidelines as well as the standard practice on PRRT in NETs $[10,19,20]$ all use $20 \%$ as the cutoff, the recently published ESMO Clinical Practice Guidelines [2] have extended the recommended upper limit of Ki-67 LI to $30 \%$. This is a grey area where considerable practice diversity exists. Adopting a dual-tracer approach could support better individualization of therapy selection in this group, where a high uptake on SRI and low FDG uptake would favor PRRT and the opposite would indicate addition of chemotherapy.

\section{Conclusion}

Here, we have taken a critical look at the potential clinical utilities of a dual-tracer imaging approach in the evaluation of patients with NET and the advantages it can offer in managing these patients. NETs are very heterogeneous, thus we believe that functional imaging may offer the important potential to biologically characterize neuroendocrine tumors such that their treatments could be better individualized and optimized. However, this concept needs to be examined more rigorously in prospective trials showing a clear benefit of the use of double-tracer imaging, and taking into consideration that international guidelines do provide different flow charts for NETs of different primaries. Their adoption alongside histopathological grading on a routine basis would hopefully enhance the scientific basis and better individualization of
Fig 4 A 54-yr-old male, diagnosed case of NET of the head of the pancreas (on biopsy, Mib $1 \mathrm{LI}$ was reported $2 \%$, serum Chromogranin A- 4,365 ng/ml).

The patient had extensive hepatic metastases as noted in the SRI and FDG-PET/CT. Both primary and hepatic metastases were heterogeneously FDG avid, with high SUVs. The patient responded poorly to PRRT and demonstrated progressive disease. Dual-tracer imaging was a correct predictor of tumor aggressiveness, though the MiB1 LI was reportedly low

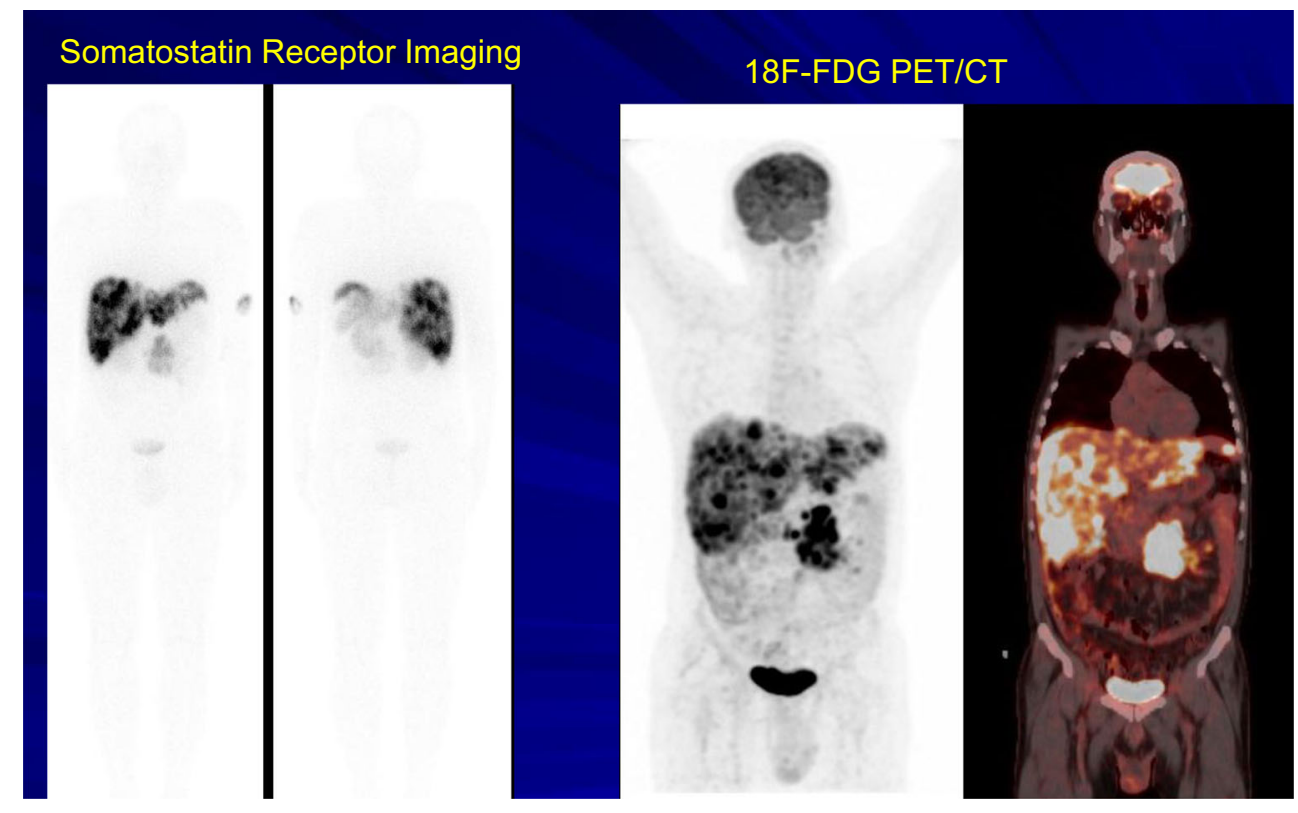


therapy, disease prognosis, and overall management of neuroendocrine tumors.

Conflict of interest The authors declare that they have no conflicts of interest.

\section{References}

1. Pavel M, Baudin E, Couvelard A, Krenning E, Öberg K, Steinmüller $\mathrm{T}$, et al. Barcelona consensus conference participants. ENETS consensus guidelines for the management of patients with liver and other distant metastases from neuroendocrine neoplasms of foregut, midgut, hindgut, and unknown primary. Neuroendocrinology. 2012;95(2):157-76.

2. Öberg K, Knigge U, Kwekkeboom D, Perren A; ESMO Guidelines Working Group. Neuroendocrine gastro-entero-pancreatic tumors: ESMO Clinical Practice Guidelines for diagnosis, treatment and follow-up. Ann Oncol. 2012 Oct;23 Suppl 7:vii124-30.

3. Klimstra DS, Modlin IR, Coppola D, Lloyd RV, Suster S. The pathologic classification of neuroendocrine tumors: a review of nomenclature, grading, and staging systems. Pancreas. 2010;39(6): 707-12.

4. Klimstra DS, Modlin IM, Adsay NV, et al. Pathology reporting of neuroendocrine tumors: application of the Delphic consensus process to the development of a minimum pathology data set. Am J Surg Pathol. 2010;34:300-13.

5. Klöppel G, Couvelard A, Perren A, et al. ENETS consensus guidelines for the standards of care in neuroendocrine tumors: towards a standardized approach to the diagnosis of gastroenteropancreatic neuroendocrine tumors and their prognostic stratification. Neuroendocrinology. 2009;90:162-6.

6. Rindi G, Kloppel G, Alhman H, et al. TNM staging of foregut (neuro) endocrine tumors: a consensus proposal including a grading system. Virchows Arch. 2006;449:395Y401.

7. Rindi G, Kloppel G, Couvelard A, et al. TNM staging of midgut and hindgut (neuro) endocrine tumors: a consensus proposal including a grading system. Virchows Arch. 2007;451:757Y762.

8. Travis WD. The concept of pulmonary neuroendocrine tumours. In: Travis WD, Brambilla E, Muller-Hermelink HK, Harris CC, eds. Pathology and Genetics of Tumours of the Lung, Pleura, Thymus and Heart. Lyon, France: IARC Press; 2004:19Y20.

9. Shrikhande SV, Sirohi B, Goel M, Barreto SG. Pancreatic neuroendocrine tumors. Indian J Gastroenterol. 2013;32(1):3-17.

10. Zaknun JJ, Bodei L, Mueller-Brand J, Pavel ME, Baum RP, Hörsch D, O'Dorisio MS, O'Dorisiol TM, Howe JR, Cremonesi M,
Kwekkeboom DJ. The joint IAEA, EANM, and SNMMI practical guidance on peptide receptor radionuclide therapy (PRRNT) in neuroendocrine tumours. Eur J Nucl Med Mol Imaging. 2013 May;40(5):800-16 (erratum: 41(3):584)

11. Basu S, Kand P, Mallia M, Korde A, Shimpi H. Gratifying clinical experience with indigeneously formulated single vial lyophilized HYNIC-TOC kit at the radiopharmaceutical division of BARC: a pivotal boost for building up a peptide receptor radionuclide therapy programme in an Indian setting. Eur J Nucl Med Mol Imaging. 2013;40(10):1622-4.

12. Turner NC, Strauss SJ, Sarker D, Gillmore R, Kirkwood A, Hackshaw A, et al. Chemotherapy with 5-fluorouracil, cisplatin and streptozocin for neuroendocrine tumours. Br J Cancer. 2010;102: 1106-12.

13. Krenning EP, de Jong M, Kooij PP, Breeman WA, Bakker WH, de Herder WW, et al. Radiolabelled somatostatin analogue(s) for peptide receptor scintigraphy and radionuclide therapy. Ann Oncol. 1999;10 Suppl 2:S23-9.

14. Kwekkeboom DJ, Krenning EP, Lebtahi R, Komminoth P, KosKudła B, de Herder WW, et al. ENETS consensus guidelines for the standards of care in neuroendocrine tumors: peptide receptor radionuclide therapy with radiolabeled somatostatin analogs. Neuroendocrinology. 2009;90:220-6.

15. Kwee TC, Basu S, Saboury B, Ambrosini V, Torigian DA, Alavi A. A new dimension of FDG-PET interpretation: assessment of tumor biology. Eur J Nucl Med Mol Imaging. 2011;38(6):1158-70.

16. Basu S, Kumar R, Rubello D, Fanti S, Alavi A. PET imaging in neuroendocrine tumors: current status and future prospects. Minerva Endocrinol. 2008;33(3):257-75.

17. Basu S, Kwee TC, Gatenby R, Saboury B, Torigian DA, Alavi A. Evolving role of molecular imaging with PET in detecting and characterizing heterogeneity of cancer tissue at the primary and metastatic sites, a plausible explanation for failed attempts to cure malignant disorders. Eur J Nucl Med Mol Imaging. 2011;38(6):98791.

18. Basu S. Should grade of tracer uptake on somatostatin receptor-targeted imaging be the major determinant and break the barrier of histopathologic criteria for determining the suitability of peptide receptor radionuclide therapy? J Nucl Med. 2013;54(11):2018-9.

19. Ezziddin S, Khalaf F, Vanezi M, Haslerud T, Mayer K, Al Zreiqat A, et al. Outcome of peptide receptor radionuclide therapy with ${ }^{177} \mathrm{Lu}$ octreotate in advanced grade $1 / 2$ pancreatic neuroendocrine tumours. Eur J Nucl Med Mol Imaging. 2014;41(5):925-33.

20. Campana D, Capurso G, Partelli S, Nori F, Panzuto F, Tamburrino D, et al. Radiolabelled somatostatin analogue treatment in gastroenteropancreatic neuroendocrine tumours: factors associated with response and suggestions for therapeutic sequence. Eur J Nucl Med Mol Imaging. 2013;40(8):1197-205. 